\title{
Somatising in children and adolescents. 1. Clinical presentations and aetiological factors
}

\author{
D. Mary Eminson
}

Somatising disorders, characterised by complaints of unexplained physical symptoms, are common presentations in children and adolescents, both in primary and secondary care settings. They have significant impact on the children themselves and on health care resources: as in adults, there is a wide range of severity and resulting handicaps.

In this paper I will examine factors that appear to predispose to such somatising disorders. The term is usually used to cover the whole group of disorders in childhood and adolescence, but here I use a narrower definition that includes only those conditions in young people in which somatic symptoms are complained of, there is insufficient or inadequate explanation in terms of a physical disorder or impairment is being caused. Other disorders, which may be somatic expressions of emotional distress, such as enuresis, encopresis and eating disorders, are not included. Common clinical presentations are described, with specific issues relevant to clinical assessment. Management and prognosis will be addressed in a subsequent paper (Eminson, 2001).

Developmental considerations are important in affecting the presentation of disorders. One developmental issue is that children and adolescents are dependants, largely reliant on adults for significant aspects of 'illness behaviour' (Mechanic, 1962). This behaviour includes the way in which symptoms are responded to, including the extent to which medical consultation and lifestyle alterations are undertaken. Parents and carers are powerful in responding to (or ignoring) children's physical complaints, attributing significance (or reassurance) to these complaints, facilitating (or otherwise) the children's use of health care facilities and their involvement in (or withdrawal from) normal life activities.

Other developmental factors, especially the level of cognitive and verbal competence, have long been recognised as important determinants of the ability to express emotional distress directly by speech. Early in development, those who are less sophisticated in their understanding and are less skilled in their direct verbal expression of psychological distress by speech are, it is suggested, more likely to express their feelings in other, more indirect ways. As psychological distress inevitably has somatic concomitants, complaints about somatic symptoms may therefore be one way in which emotional distress is both experienced and communicated by young people, just as for young children a variety of other primitive and regressive behaviours are commonly recognised as signalling emotional discomfort of various kinds. It is assumed that the process itself, of somatising emotional distress, is a common if not ubiquitous experience in childhood. This is not to suggest that just being a child can, in itself, be sufficient to cause the extent of somatising presentations in childhood and adolescence, the different forms of distress seen clinically or the natural history of these disorders. A variety of other explanations are relevant and must be sought, including temperamental, psychosocial, cultural and possibly genetic factors.

D. Mary Eminson is a consultant child and adolescent psychiatrist at Bolton Hospitals NHS Trust, Royal Bolton Hospital, Minerva Road, Bolton BL4 OJR (tel.: 01204 390663; fax: 01204 390660; e-mail: Mary.Eminson@boltonh-tr.nwest.nhs.uk). She has a long-standing clinical and academic interest in all aspects of abnormal illness behaviour in children and parents, and in parents who fabricate illness in their children. 


\section{Classification}

It is perhaps not surprising that the forms of somatising disorders found during the course of childhood and adolescence are not uniform and that the clinical patterns of presentation in adolescence bear a much greater resemblance to adult disorders than is the case in earlier childhood. Box 1 summarises the disorders. Using the ICD-10 classification (World Health Organization, 1996), only two categories are specific to the developmental stage: adjustment reactions and transient dissociative disorders of childhood. If not placed in either of these categories, children and adolescents must be accommodated within the adult categories, even though the terminology and symptom description are ill-suited to a childhood population. In many respects, this section of ICD-10 is ripe for radical review of language and conceptualisation, to include awareness of differences in presentation throughout development. However, despite its limitations it has been broadly adhered to

Box 1 Major diagnostic categories for somatising disorders in children and adolescents

Adjustment disorders - mixtures of psychological symptoms (anxiety, temper and irritability) and regressive behaviours such as thumb sucking and bedwetting (which may include physical symptoms) arising in response to life events or stresses. The physical symptoms are typically headaches, stomach and joint pains, but may be in any system and multiple.

Dissociative (conversion) disorders (includes transient dissociative disorders) - losses of function, mainly movement or sensation: blindness, deafness, pseudo-seizures, paralysis and losses of sensation are the most common presentations in childhood and adolescence.

Somatoform disorders - various presentations in which different symptoms predominate, and with three levels of chronicity. Common characteristics are repeated presentation of physical symptoms with requests for medical investigations, despite doctors' failure to find clinical symptoms and consequent reassurances that the symptoms have no physical basis. Any physical findings do not explain the nature and extent of the symptoms and distress.

Categories include:

Somatisation disorder -2 years of multiple, variable symptoms

Undifferentiated somatoform disorder -6 months of multiple, variable symptoms with less severity and handicap than somatisation disorder

Hypochondriacal disorder - persistent preoccupation with, and belief in, the presence of at least one serious physical illness

Persistent somatoform pain disorder - persistent, severe, distressing pain that is more persistent and dominant than the multiple aches and pains of other categories

Other somatoform disorders - physical complaints limited to specific systems or parts of the body, and disorders of sensation such as tingling and numbness. There is no minimum time-scale for the complaints

Chronic fatigue syndrome (CFS) (neurasthenia) - criteria adopted by the Association for Child Psychology and Psychiatry Working Group (and adapted from those for adults) have largely replaced the ICD-10 neurasthenia categories.

Criteria include:

Disabling fatigue, affecting physical and mental functioning (the cardinal symptom)

Other symptoms, including aches and pains, headaches, sleep disturbance and concentration difficulties

A definite onset for the syndrome, which may or may not follow a viral illness course

A minimum duration of 6 months, although treatment may need to begin sooner

Anxiety and/or depressive symptoms may be present and do not prevent diagnosis (other psychiatric disorders, such as anorexia nervosa or a depressive disorder, should be distinguished from CFS)

Factitious disorders: intentional production or feigning of symptoms or disabilites, either physical or psychological; typical presentations are skin lesions, damage to eye lashes or cornea or damage to exisiting wounds preventing their healing 
and further critique is outside the scope of this paper.

The somatic disorder categories are currently based on the number, nature, chronicity and impact of the unexplained symptoms. In brief, this produces four major categories: dissociative disorders, somatoform disorders, chronic fatigue syndrome (a term that has generally replaced neurasthenia) and factitious disorders.

For completeness, a fifth category, adjustment disorder, is included in this review, because it is the most accurate description for many short-lived, single- or multiple-symptom presentations in childhood and early adolescence that have obvious precipitants and are usually seen and managed in paediatric clinics or in primary care.

The inclusion of factitious presentations may be seen as controversial, but it is here for both pragmatic and theoretical reasons. First, clinically, they fit the criteria for somatising disorders because they are characterised by a somatic presenting complaint, with no physical disorder explanation. They are presented in a medical arena and cause impairment. Second, there are similarities too in terms of aetiology, as many factitious disorders may be viewed as maladaptive ways of expressing distress somatically. Third, the distinction between factitious and non-factitious symptoms is often almost impossible in practice and it is already recognised that there are factitious elements in many of the somatising groups. Finally, the principles of management are similar for both types of disorder.

\section{Background}

The aetiology of childhood somatising disorders is complicated by the difficulty of distinguishing factors relevant to the experience and complaint of physical symptoms from those that contribute to the various aspects of illness behaviour. Biological, psychological and social factors are relevant to both the complaints and the illness-behaviour responses to them. Thus, the aetiology may be very different for two children with similar symptoms, leading to problems in generalising. Although more sophisticated research is being undertaken that may demonstrate robust associations between specific disorders and predisposing risk factors, in the current state of knowledge it is best to view childhood somatising disorders as being reached via different 'pathways', there being a wide range of precipitating and maintaining factors, in both child and family, that may result in a clinically significant presentation, sufficient to reach criteria for a disorder. The corollary of this is that none of the individual factors (outlined in Box 2) are, on their own, either necessary or sufficient for such a presentation: a combination of vulnerability and trigger factors will be maintained by issues unique to each child and family. This point is emphasised because in the past there was too readily adherence to the assumption that all children or families with a particular presentation 'must' possess certain risk

Box 2 Somatising disorders

Predisposing factors

Family:

Many somatic symptoms experienced (genetic component?)

Limited in verbal communication about emotional issues, including conflict

'Conditional caretaking'

Suspicious attitude to medical expertise

Parental history of somatoform illness, anxiety or depression

Problems with boundary setting for children

Child:

Temperamental factors, including conscientiousness, emotional lability, vulnerability and worthlessness

Earlier emotional abuse

Low IQ

Social-relating difficulties

Precipitating factors

Child:

Anxiety, depression

Life stresses of all types - overt and covert

Physical illness

Peer group problems

Academic problems and cognitive limitations

Low self-esteem

Parent:

Life events/crises

Maintaining factors

Child, ${ }^{*}$ parent and professional:

Current family relationship difficulties

*Predicament is resolved by symptoms

Family model of serious illness

Current parental mental ill health, particularly anxiety and somatisation

*School problems

Models of sickness and conflict avoidance

Benefits of sick role

Professional behaviour that reinforces anxieties and sick role 
factors; for example, that major family dysfunction must be a feature of somatoform presentations with a highly symptomatic child.

\section{Factors associated with reporting physical symptoms}

Numerous epidemiological studies, predominantly of adolescents and large clinical samples that include younger children, have been used to examine reports of unexplained symptoms and their correlates. These studies are consistent in their findings about what increases the likelihood of physical symptoms in the population and factors that are likely, in combination, to be a sufficient explanation of many mild to moderate adjustment and conversion disorders. These factors could perhaps also be significant in the other major disorders.

Gender is consistently reported to be associated with differential reporting: girls experience more symptoms than boys, and girls report more symptoms as adolescence proceeds (Aro, 1987). In the majority of studies, girls have been found to report symptoms at increasing rates during adolescence, while reporting levels by boys fall during this time. Therefore, with increasing age it seems that boys attend to, and consistently report, fewer physical symptoms (Aro, 1987; King \& Coles, 1992). Presumably, cultural factors in the socialisation of the different genders are also relevant. In some, but not all, studies the onset of pubertal development and the menarche is associated with increased symptom reporting in girls (Aro \& Taipale, 1987).

Within any one age band, the factors that increase the likelihood of reporting more symptoms are also consistent across studies. Pre-eminent among these factors are psychological conditions, perhaps most notably anxiety and depression (Egger et al, 1999). In young children (aged 4-6) difficult behaviour is also associated with more symptom complaints (Faull \& Nicol, 1986). Temperamental factors in children have also been identified as relevant, with characteristics such as anxiety traits, perfectionism and conscientiousness being reported in many clinical studies. Both general poor school performance and poor attainment relative to teachers' perceptions of the adolescent's ability are associated with more symptoms (Eminson et al, 1996). In several studies those who perceived their self-competence as low have been found to have high levels of symptoms. Chronically stressful social and family situations such as parental disharmony increase symptoms, as do acute life stresses (change of school, acute family problems).
It has been suggested that genetic factors may play a part in sensitivity to pain and other bodily sensations, although little hard evidence exists (Benjamin \& Eminson, 1992). Disrupted earlier life experience and poor parental care (including abuse, neglect and 'conditional caretaking' - attending to children when they complain of physical symptoms but not when they make other types of request, e.g. for affection and care) are also associated with increased symptom reports and are found in the histories of adults with excessive unexplained symptoms (Craig et al, 1993). A consistent finding of these studies is that questionnaires identify those adolescents with a general tendency to report symptoms. It is this trend to report physical symptoms that is consistently associated with other markers of disturbance and distress. Family factors have an influence on the level of children's symptoms - if parents have physical symptoms they are more likely to have children with increased rates of symptoms. However, parents' relative ignorance of their children's physical symptoms has also been demonstrated (Taylor et al, 1996), with many 'false negatives' in parental reports.

\section{Factors affecting illness behaviour}

\section{Children's contributions to illness behaviour}

Children themselves, even at a young age, are far from insignificant contributors to illness behaviour, despite the relative importance of parents in decisions about how children's complaints are managed. In descriptive studies, children from a very early age ( 3 or 4 years) display an understanding of 'illness behaviour', that is to say they appreciate that expectations of behaviour are different in those who are sick and that by displaying certain behaviour, one can signal to others that one is sick and should be relieved of normal tasks (Wilkinson, 1988). Thus, an understanding of the sick role is readily acquired by children as part of family life, although this will be heavily influenced by parental models. Older children are able to display independently a wide range of illness behaviours.

\section{Family and parental factors}

Boundary setting and beliefs

Parents or caregivers are the ultimate decisionmakers about the responses to a child's symptoms 
and illness behaviour, and they decide whether a child's complaints are sufficiently severe to allow him or her to be judged to be sick, and if so, whether he or she should be treated as sick by missing school, being given medicine or taken to a health professional. Most parents find it relatively easy to decide when a physical illness has come to an end and when to support and encourage the child to leave the home and resume a normal life by going back to school. Such parents will explore and resolve with the child and teachers the reasons behind evident reluctance. But for some other parents, who are conscientious and caring and may have no parenting difficulties in other ways, these decision-making processes become difficult, resulting in problems encouraging a child to ignore his or her symptoms and return to ordinary activities. The reasons for this are often related to early parenting experiences or aspects of the parent-child relationship that were not readily established, but can be powerful. It has been demonstrated (McGrath, 1995) that the maternal attitude to pain has an effect on the length of time children are away from school following illness. Similarly, Rangel \& Garralda (2000) have demonstrated that parental belief in a physical cause for a child's chronic fatigue symptoms is associated with a poorer prognosis at follow-up.

\section{Parental ill health}

Parental ill health (mental or physical) may be associated with a difficulty in believing a child is healthy, or has only minor illness, and anxiety about the child's health, with a distorted or pessimistic analysis of the situation. Parental depression and anxiety increase the likelihood of consultation by parents for both themselves and for their children in primary and secondary care (Garralda \& Bailey, $1987,1989)$. In studies of paediatric consulters and of epidemiology, it has been shown that parents with more physical and mental health problems are more likely to have children with functional somatic symptoms than are parents without such complaints (Hotopf et al, 1998).

\section{Emotional expression}

It has long been suggested, and there is some limited evidence, that independent of the other individual cognitive, social, cultural and psychological factors, individuals differ in their capacity to express emotions directly, and that to some extent this is learned within families. This is the basis of concepts such as 'psychological mindedness' and its almost opposite, 'alexithymia' (literally meaning no word for feelings) (Sifneos, 1973). The latter term is used in case reports to describe families with severe difficulty in emotional expression where a child has presented with a somatoform disorder (Kraemer \& Loader, 1996). These suggestions may help to explain why physical complaints are an easier route to expressing distress in some families. Another way these ideas have been expressed has been in the notion of 'conditional caretaking', that is the suggestion that some parents may respond differentially to physical and emotional distress, with preferential responses to the former, which the child quickly learns. The reasons why some parents find it difficult to hear or heed emotional cues or language, or respond in kind, are not fully understood and research into this is awaited.

One route already identified as resulting in limited emotional expression combined with functional somatic complaints is early emotional and physical abuse, although the mechanisms are far from clear (Craig et al, 1993). Certainly, in clinical practice children and adolescents, with multiple symptoms who come from emotionally deprived and neglectful backgrounds are commonplace, and the function of symptoms as primitive markers of distress is often easy to recognise. Dissociation from painful emotional experiences in early life, retaining only an awareness of physical distress, is one possible mechanism.

\section{Cultural factors}

Cultural factors will affect beliefs about symptoms and the seriousness attached to them, as will topical illnesses (currently including 'myalgic encephalomyelitis' (ME) and concerns about allergens, for example). Attitudes to the medical profession, to health and to the benefits or otherwise of normal school and social activities obviously vary widely and have powerful shaping effects on parents and children. These are variations within prevailing cultural norms and families from other cultural and ethnic backgrounds will have a wider range of beliefs and conventions about illnesses and about ways in which psychological distress may be organised somatically.

\section{Clinical presentations}

\section{Adjustment reactions}

This is probably the most accurate psychiatric label for the common, short-lived or sometimes recurrent symptoms presented to general practitioners or paediatricians, who often exclusively manage the patient. Developmentally, these are the first 
disorders to be seen and are as common in boys as girls. Characteristically beginning in early and middle childhood, stomach aches, headaches, joint pains or tiredness are the focus of recurrent complaints that cause parents to seek medical advice. They result in the child missing school and limiting social activities. Although these presentations are so common that to make generalisations about background factors is only broadly applicable, factors contributing to such presentations often include anxiety, and, in younger children, oppositionality and behavioural disturbance (Faull \& Nicol, 1986). The children are often somewhat shy and compliant outside the home and somatic complaints are used as an expression of distress, usually in a long-standing pattern, often with family traits of anxiety, protectiveness and somatisation.

\section{Transient dissociative disorders}

\section{Dissociative disorders}

These are disorders that are characterised by a loss of function in any modality and appear from middle childhood onwards (Goodyer \& Taylor, 1985; Grattan-Smith et al, 1988). The best known for young people is apparent loss of motor function. Complete loss of sight, hearing, sensation and consciousness (as in a pseudo-seizure) or partial loss (as in a fugue state) are also reported, although fugue states are uncommon in this age group. The true prevalence of either transient or more long-lasting losses of function is unknown, partly because the most transient disorders are not necessarily brought to medical attention or, if taken to an accident and emergency department or other clinic, will often remit quickly with reassurance. But these are not uncommon disorders and only prolonged reactions are likely to be presented in a mental health arena.

The capacity for experiencing unexplained symptoms or losses of function is extremely widespread in young people - in girls particularly - although outside 'epidemic' presentations there is little difference between the genders in numbers presenting. In its epidemic forms, it is generally known as mass hysteria or mass sociogenic illness, is often reported in schools or workplaces and is usually focused on topical fears (currently, allergens and environmental toxins). Having no evidence of psychological disturbance, most sufferers make a speedy recovery. Many aetiological explanations have been advanced to explain these losses of function. Those whose symptoms persist beyond the few days of the epidemic will usually be found to be more vulnerable, and more of the risk factors for somatoform disorders will be found in their background information.
Wynick et al (1997), in studying psychogenic disorders of vision in childhood (a variety of symptoms including blindness and blurred vision), found difficulties at school, significant losses, adjustment difficulties and some obsessional personality traits in the adolescents they studied. In addition, parental overprotectiveness was reported by adolescents and largely acknowledged by parents. Such combinations of difficulties are characteristic; learning difficulties and peer group problems are extremely prevalent; and finding a number of predisposing risk factors with a few 'triggering' issues is more common than identifying a frank psychiatric diagnosis such as depression. Comparisons were with other adolescents with visual problems of organic origin.

\section{Somatoform disorders}

This category, which includes somatisation disorder, the more short-lived undifferentiated somatoform disorder and various sub-categories, includes uncommon, but far from rare, disorders usually seen in paediatric clinics and appearing first in middle childhood then more commonly in adolescence. Unfortunately, the clinical examples in ICD-10 (World Health Organization, 1996) are atypical of childhood and adolescence; for example, genitourinary symptoms are rare. Nevertheless, these patients will be found attending most paediatric specialities, often with gastrointestinal complaints, joint and other pains and neurological symptoms. More girls than boys present in this way and, even by mid-adolescence, there may be a severe picture of increasingly poor school attendance and attainment and social withdrawal. Even the milder forms include young people with a range of personal and family background vulnerabilities, and may also include body image symptoms, with self-harm and eating problems.

However, in the more severe and chronic adolescent presentations there is a proportion with somatic symptoms which are sometimes combined with other, more conduct-disorder difficulties and the predisposing factors clearly include more severe and earlier problems, attachment difficulties and sometimes emotional, physical or sexual abuse. Major family dysfunction is often present, with recent obvious psychosocial stresses. Family disorganisation may include breakdown and criminality. There may have been a long history of less extensive physical complaints and abnormal illness behaviour, usually with patchy school attendance together with a family history of both organic physical illness and marked illness behaviour. The extent to which lifestyles are altered by the 
somatising disorder varies widely, as does the tendency to consult medical facilities and seek investigations, and may also vary in any individuals at different times. Often the young person and his or her family demonstrate indifference or antagonism to psychological or psychiatric help and there is neglect and failure to attend follow-up for concurrent organic physical conditions. Educational failure almost invariably ensues. These presentations occur where social deprivation is closely juxtaposed to medical facilities, but no adequate estimates of prevalence in clinical populations exist.

\section{Hypochondriacal disorder}

Virtually unknown before adolescence, and uncommon during it, hypochondriasis appears to be the last somatoform disorder to develop clinically, characterised by an unshakeable belief in the presence of an illness or disease. However, body dysmorphic disorders, included in this category for ICD-10 (but distinguished in DSM-IV), are more common, making their mark from mid-adolescence onwards, but with much overlap with eating disorders. Lack of standardised instruments or operationally defined criteria for hypochondriasis are likely to slow the identification of any such disorders in adolescent medicine.

\section{Pain disorders (persistent somatoform pain disorders)}

It has long been suggested that the separation of pain from other symptoms in paediatric studies of somatisation is unhelpful (McGrath, 1993) because there is substantial overlap with the other categories of somatoform disorder outlined earlier and occurring in middle childhood and adolescence, usually presenting through secondary paediatric or orthopaedic care. Some children who, when younger, were described as having adjustment disorders (e.g. headaches or abdominal pain) also reach criteria for this disorder in adolescence, but rarely before. Pain clinics may contain a number of sufferers (and incidentally may also provide an acceptable way to involve mental health professionals through focusing initially on symptom relief) but, as with the other somatoform disorders, reliable prevalence estimates are lacking. There are no studies that distinguish specific aetiological factors from other 'somatoform' disorders. There is a similar (small) proportion of patients with frank psychological symptoms (usually depression, often thought by the patient to be secondary to his or her pain and somatic complaints). Parental responses to symptoms are crucial (McGrath, 1993). Perhaps the typical patient is an adolescent girl with pain in a limb, which is therefore unused and may show typical secondary changes as a result. The term 'reflex sympathetic dystrophy' may be used by rheumatologists or orthopaedic surgeons to describe a condition of uncertain aetiology, sometimes with some organic components, but where psychological factors play a significant role in the illness (Murray et al, 2000). If enquired after carefully, many other symptoms, including fatigue, can be uncovered.

\section{Chronic fatigue syndrome}

Disabling fatigue has long been recognised as a troublesome condition (or group of conditions) that affects girls more than boys from middle childhood onwards and has many different diagnostic labels. There is wide speculation about possible aetiologies, including immunological, nutritional and other hypotheses. While fatigue is by definition the most prominent among the physical symptoms (Box 1), there are usually many others and sleep disturbance and eating problems are common. The most seriously affected children and adolescents may be confined to a wheelchair and unable to take any part in normal life for many years. This level of disturbance has been called 'pervasive refusal' and is recognised to have considerable overlap with severe eating disorders. In most studies girls predominate, which is common to all adolescent somatising disorders. Family factors associated with the condition in childhood have recently been reviewed by Pipe (1999), in a useful collection of papers devoted to chronic fatigue (Garralda, 1999). Major family factors include family illness, both physical and psychological closeness, protectiveness and difficulty with boundary setting. In one of the few studies on childhood, using standardised measures, Rangel et al (1999) found that sufferers were more likely than controls to have personality difficulty or disorder, the common personality features being conscientiousness, vulnerability, worthlessness and emotional lability.

\section{Factitious disorders}

Young people with factitious disorders, also commonly girls, present to all paediatric specialists from middle childhood onwards (Libow, 2000). Existing lesions (skin rashes, sutures) are interfered with to produce more pathology, or lesions are produced by picking at the skin or the eye. Such lesions are incontrovertibly produced by the child or adolescent 
him- or herself. Sometimes there is quite obvious 'play acting', for example make-up applied to simulate cellulitis has been reported (King \& Chalmers, 1984). Some children have a single symptom; many seem to have an intense desire for medical attention, in active pursuit of a sick 'role'. There is no uniformity in the severity of the young person's or family's disturbance and some straightforward presentations are readily understood as an unsophisticated communication to resolve a difficult predicament (Taylor, 1982), whereas others are much less easy to explain. Some presentations, both acute and chronic, are accompanied by other somatic symptoms or somatising disorders, with which there is substantial overlap. The more extensive and tenaciously pursued fabrications are more likely to indicate significant major difficulties in personal relationships, often with a superficial and immature relating style but without overt psychiatric symptoms. In these most severe cases difficult temperamental traits may be traced to very early disturbance in relationships, with disrupted attachments, sometimes including extensive physical, emotional and sexual abuse, very similar to the picture described earlier in some severe somatisation disorders. Others in intact families may not have an abusive background but may have few personal resources and a predicament or dilemma (e.g. major school failure and unpopularity; current extra-familial sexual abuse) that is resolved (by the results of the fabrication), or to which attention is drawn by the fabrication.

\section{Summary}

The clinical presentations of somatising disorders in childhood and adolescence begin in early childhood but the more seriously handicapping disorders are usually evident from middle childhood and, even more commonly, in adolescence. Biological, psychological and social factors are relevant to both the experience and report of unexplained somatic complaints, and to the ways in which the individuals and their carers respond to these complaints. Five major forms of presentation have been described and aetiological factors relevant to these outlined. Estimates of prevalence are not offered, because of the paucity of studies including physical and mental health specialists and primary care.

\section{References}

Aro, H. (1987) Life stress and psychosomatic symptoms among 14-16 year old Finnish adolescents. Psychological Medicine, 17, 191-201.
— \& Taipale, V. (1987) The impact of timing of puberty on psychosomatic symptoms among fourteen to sixteen year old Finnish girls. Child Development, 58, 261-268.

Benjamin, S. \& Eminson, D. M. (1992) Abnormal illness behaviour: childhood experiences and long-term consequences. International Review of Psychiatry, 4, 55-70.

Craig, T. K. J., Boardman, A. P., Mills, K., et al (1993) The South London somatisation study. I: Longitudinal course and the influence of early life experiences. British Journal of Psychiatry, 163, 579-588.

Egger, H. L., Costello, E. J., Erkanli, A., et al (1999) Somatic complaints and psychopathology in children and adolescents: stomach aches, muscular-skeletal pains and headaches. Journal of American Academy of Child and Adolescent Psychiatry, 38, 852-860.

Eminson, D. M. (2001) Somatising in children and adolescents. 2. Management and outcomes. Advances in Psychiatric Treatment, 7, in press.

--, Benjamin, S., Shortall, A., et al (1996) Physical symptoms and illness attitudes in adolescents: an epidemiological study. Journal of Child Psychology and Psychiatry, 37, 519-527.

Faull, C. \& Nicol, A. R. (1986) Abdominal pain in six-yearolds: an epidemiological study in a new town. Journal of Child Psychology and Psychiatry, 27, 251-260.

Garralda, E. (1999) Chronic Fatigue Syndrome: Helping Children and Adolescents. Occasional Papers OP16. Oxford \& New York, NY: Association for Child Psychology and Psychiatry.

— \& Bailey, D. (1987) Psychosomatic aspects of children's consultations in primary care. European Child and Adolescent Psychiatry, 236, 319-321.

— \& - (1989) Psychiatric disorders in general pediatric referrals. Archives of Disease in Childhood, 46, 1727-1733.

Goodyer, I. \& Taylor, D. C. (1985) Hysteria. Archives of Disease in Childhood, 60, 680-681.

Grattan-Smith, P., Fairley, M. \& Procopis, P. (1988) Clinical features of conversion disorder. Archives of Disease in Childhood, 63, 408-414.

Hotopf, M., Carr, S., Mayou, R., et al (1998) Why do children have chronic abdominal pain, and what happens to them when they grow up? British Medical Journal, 316, 1196-1200.

King, A. J. \& Coles, B. (1992) The Health of Canada's Youth: Views and Behaviours of 11-, 13- and 15-Year Olds from 11 Countries. Canada: Minister of National Health and Welfare.

King, C. M. \& Chalmers, R. J. (1984) Another aspect of contrived disease: "dermatitis simulata". Cutis, 34, 463464

Kraemer, S. \& Loader, P. (1996) Passing Through Life: Alexithymia and Attachment Disorders. Occasional Paper OP12. Oxford \& New York, NY: Association for Child Psychology and Psychiatry.

Libow, J. A. (2000) Child and adolescent illness falsification. Pediatrics, 105, 336-342.

Mechanic, D. (1962) The concept of illness behaviour. Journal of Chronic Disorders, 15, 189-194.

McGrath, P. J. (1993) Pain in Adolescents (eds N. L. Schechter, C. B. Berde \& M. Yaster). London: Williams and Wilkins. - (1995) Aspects of pain in children and adolescents. Journal of Child Psychology and Psychiatry, 36, 717-730.

Murray, C. S., Cohen, A., Perkins, T., et al (2000) Morbidity in reflex sympathetic dystrophy. Archives of Disease in Childhood, 82, 231-233.

Pipe, R. (1999) A Review of Family Factors in Chronic Fatigue Syndrome. Occasional Paper OP16 (ed. E. Garralda), pp. 9-12. Oxford \& New York, NY: Association for Child Psychology and Psychiatry

Rangel, L. \& Garralda, E. (2000) Personality in adolescents with chronic fatigue syndrome. European Child and Adolescent Psychiatry, 9, 39-45.

—, - - E., Levin, M., et al (1999) Psychiatric adjustment in adolescents with a history of chronic fatigue syndrome. Journal of the American Academy of Child and Adolescent Psychiatry, 38, 1515-1521.

Sifneos, P. (1973) The prevalence of 'alexithymic' characteristics in psychosomatic patients. Psychotherapy and Psychosomatics, 22, 255-262. 
Taylor, D. C. (1982) The components of sickness: disease, illnesses and predicament. In One Child (eds J. Apley \& C. Ounsted), pp. 1-13. London: Spastics International Medical Publications.

_- Szatmari, P., Boyle, M., et al (1996) Somatization and the vocabulary of everyday bodily experiences and health concerns: a community study of adolescents. Journal of the American Academy of Child and Adolescent Psychiatry, 35, 491-499.

Wilkinson, S. R. (1988) The Child's World of Illness: The Development of Health and Illness Behaviour. Cambridge: Cambridge University Press.

World Health Organization (1996) Multiaxial Classification of Child and Adolescent Psychiatric Disorders. The ICD-10 Classification of Mental and Behavioural Disorders in Children and Adolescents. Cambridge: Cambridge University Press.

Wynick, S., Hobson, R. P. \& Jones, R. B. (1997) Psychogenic disorders of vision in childhood ("visual conversion reactions"): perspectives from adolescence. A research note. Journal of Child Psychology and Psychiatry, 38, 375379.

\section{Multiple choice questions}

1. Dissociative disorders of childhood and adolescence:

a of both transient and chronic type occur more commonly in girls

b when transient, often respond well to reassurance alone

c when prolonged beyond a few weeks, usually share many characteristics with other 'somatising' disorders

e are associated with school presentations and learning problems

$\mathrm{f}$ are commonly presented with fugue states in adolescents.

2. The development of somatising disorders during childhood demonstrates that:

a persistent somatoform pain disorder is developmentally the last to appear

b somatising disorders may be preceded by adjustment reactions with prominent physical symptoms

c characteristic presenting complaints for ICD10 somatoform disorders are very similar in children and adults

d headaches, stomach-aches and joint pains are the most common early symptoms

e hypochondriacal disorder is not unusual in middle childhood.
3. An increased likelihood of reporting somatic symptoms in childhood (pre-adolescence) is associated with:

a difficult behaviour

b negative-impact life events

c anxiety traits

d conscientious and perfectionist temperamental traits

e above average school achievements in most cases.

4. Family factors associated with childhood somatising disorders include:

a parents with an excess of physical symptoms

b parents with depression and anxiety showing reduced rates of consultation for their children's symptoms

c the length of time the child suffers impairment being affected by maternal beliefs about the child's illness

d characteristic patterns of family functioning

e intrafamilial emotional and physical neglect and abuse.

5. Factitious disorders in childhood and adolescence:

a are almost always associated with severe child psychopathology

b are not characterised by parents' fabrication of symptoms in adolescents

c are best assessed as a form of somatising disorder

d present commonly to ophthalmologists and dermatologists

e may be found in conjunction with another somatising disorder.

\begin{tabular}{|c|c|c|c|c|c|}
\hline \multicolumn{6}{|c|}{ MCQ answers } \\
\hline 1 & 2 & 3 & 4 & & 5 \\
\hline a $F$ & a $F$ & a $T$ & & $\mathbf{T}$ & \\
\hline b $\mathbf{T}$ & b $\mathbf{T}$ & b T & & $\mathbf{F}$ & b F \\
\hline$c T$ & c $\mathbf{F}$ & c $T$ & & $\mathbf{T}$ & c $T$ \\
\hline $\mathbf{T}$ & d $T$ & d $T$ & d & $\mathbf{F}$ & d $T$ \\
\hline $\mathbf{F}$ & e $F$ & e $\mathbf{F}$ & & $\mathbf{F}$ & e $\mathrm{T}$ \\
\hline
\end{tabular}

